$10-1-2010$

\title{
Leg ulcers in sickle cell disease.
}

\author{
Caterina P Minniti \\ National Institutes of Health \\ James Eckman \\ Emory University School of Medicine
}

Paola Sebastiani

Boston University School of Public Health

Martin H Steinberg

Boston University School of Medicine

Samir K. Ballas

Thomas Jefferson University

Follow this and additional works at: https://jdc.jefferson.edu/medfp

Part of the Medical Genetics Commons

Let us know how access to this document benefits you

\section{Recommended Citation}

Minniti, Caterina P; Eckman, James; Sebastiani, Paola; Steinberg, Martin H; and Ballas, Samir K., "Leg ulcers in sickle cell disease." (2010). Department of Medicine Faculty Papers. Paper 50. https://jdc.jefferson.edu/medfp/50

This Article is brought to you for free and open access by the Jefferson Digital Commons. The Jefferson Digital Commons is a service of Thomas Jefferson University's Center for Teaching and Learning (CTL). The Commons is a showcase for Jefferson books and journals, peer-reviewed scholarly publications, unique historical collections from the University archives, and teaching tools. The Jefferson Digital Commons allows researchers and interested readers anywhere in the world to learn about and keep up to date with Jefferson scholarship. This article has been accepted for inclusion in Department of Medicine Faculty Papers by an authorized administrator of the Jefferson Digital Commons. For more information, please contact: JeffersonDigitalCommons@jefferson.edu. 


\title{
As submitted to:
}

\section{American Journal of Hematology}

\author{
And later published as:
}

\section{"Leg Ulcers in Sickle Cell Disease”}

\section{Volume 85, Issue 10, October 2010, Pages 831-833}

\section{DOI: 10.1002/ajh.21838}

Caterina P. Minniti ${ }^{1,}{ }^{*}$, James Eckman ${ }^{2}$, Paola Sebastiani ${ }^{3}$, Martin H. Steinberg ${ }^{4}$, Samir K. Ballas ${ }^{5}$

Sickle cell disease is a single amino acid molecular disorder of hemoglobin leading to its pathological polymerization, red cell rigidity that causes poor microvascular blood flow, with consequent tissue ischemia and infarction. The manifestations of this disease are protean. Among them, leg ulcers represent a particularly disabling and chronic complication, often associated with a more severe clinical course. Despite the fact that this complication has been recognized since the early times of SCD, there has been little improvement in the efficacy of its management and clinical outcome over the past 100 years. Recently, vasculopathic abnormalities involving abnormal vascular tone and activated, adhesive endothelium have been recognized as another pathway to end organ damage in sickle cell disease. Vasculopathy of sickle cell disease has been implicated in the development of pulmonary hypertension, stroke, leg ulceration and priapism, particularly associated with hemolytic severity, and reported in other severe hemolytic disorders. The authors present the proceedings from the Educational Session on Chronic leg ulcers in Sickle cell disease, held during the 4th Annual Sickle Cell Disease Research and Educational Symposium, on February 17, 2010 in Fort Lauderdale, Fla.

The clinical manifestations of sickle cell disease (SCD) are diverse. Among them, leg ulcers are relatively common and can be disabling. Leg ulcerations have been a long recognized complication of SCD and even the first SCD patient to be described in North America in 1910 had chronic leg ulcerations [1, 2]. The prevalence of leg ulceration varies, being low before age 10 years. It is most common in sickle cell anemia (homozygosity for HBB glu6val; HbSS) and less often seen in $\mathrm{HbSC}$ disease or $\mathrm{HbS}-\beta$ thalassemia. Its geographical distribution is also variable, affecting $75 \%$ of $\mathrm{HbSS}$ patients in Jamaica but only $8-10 \%$ of North American patients [3-5]. The pathogenesis of chronic 
ulcers in SCD is complex. Mechanical obstruction by dense sickled red cells, venous incompetence, bacterial infections, abnormal autonomic control with excessive vasoconstriction when in the dependent position, in situ thrombosis, anemia with decrease in oxygen carrying capacity, and decreased nitric oxide bioavailability leading to impaired endothelial function have all been proposed as potential contributing factors $[6,7]$.

Venographic studies have shown that venous insufficiency is not a primary cause of sickle cell ulcerations. Instead, it is the arteriovenous shunting, which has been postulated as a factor in ulcer formation. This shunting deprives the skin of oxygen promoting ulceration. Furthermore, trauma, infection, and inflammation have all been cited as potential causes of lower extremity ulcerations in SCD. It is the combination of these factors, along with the interrupted microcirculation that contribute to the pathophysiology of lower extremity ulcerations in patients with SCD.

Leg ulcers occur in areas with less subcutaneous fat, thin skin, and with decreased blood flow [8]. The commonest sites are the medial and lateral malleoli (ankles), often becoming circumferential if not controlled early; the medial malleolus is more commonly involved than the lateral malleolus. Less common sites are the anterior tibial area, dorsum of the foot, and Achilles tendon [9].

\section{Severity Index}

Many severity indices for leg ulcers are based on size, depth, and duration. Staging based on depth is as follows:

Stage 1: Nonblanchable erythema of intact skin, the heralding lesion of skin ulceration. In individuals with darker skin, discoloration of the skin, warmth, edema, induration, or hardness may also be indicators.

Stage 2: Partial-thickness skin loss involving epidermis, dermis, or both. The ulcer is superficial and presents clinically as an abrasion, blister, or shallow crater.

Stage 3: Full-thickness skin loss involving damage to or necrosis of subcutaneous tissue that may extend down to, but not through, underlying fascia. The ulcer presents clinically as a deep crater with or without undermining of adjacent tissue.

Stage 4: Full-thickness skin loss with extensive destruction, tissue necrosis, or damage to muscle, bone, or supporting structures (e.g., tendon and joint capsule). Undermining and sinus tracts also may be present.

\section{Classification}

Leg ulcers may be classified as acute or chronic according to their duration, however, there is no consensus as to a specific length of time to define chronicity. An acute ulcer usually heals in less than a month. Among chronic ulcers, duration of 6 months seems to define the most recalcitrant ulcers. It is not uncommon for ulcers to last many years, often closing and reopening repeated times.

\section{Clinical Features}


Leg ulcers are painful and often. They tend to be indolent, intractable, and heal slowly over months to years. The pain may be severe, excruciating, penetrating, sharp, and stinging in nature. In most patients, oral or parenteral opioid analgesics are needed to achieve some pain relief. Leg ulcers are more common in males in some studies and their incidence increases with age. Their association with the severity of hemolysis is suggested by their reduced incidence in carriers of coincident $\alpha$-thalassemia, individuals with higher hemoglobin concentrations and those with high levels of $\mathrm{HbF}[5]$. They are also associated with priapism and pulmonary hypertension, both felt to be markers of advanced sickle vasculopathy. Moreover, leg ulcers have been reported to be more common in carriers of the CAR $\beta$ globin gene cluster haplotype.

\section{Management}

Treatment of leg ulcers includes wound care using wet to dry dressings. With regular and good localized treatment, many small ulcers may heal within a few months. Leg ulcers that persist beyond 6 months may require other modalities including, among other things, blood transfusion, skin grafting, Unna boots, zinc sulphate, hyperbaric oxygen, arginine butyrate, topical herbal applications, topical growth factors. Unfortunately, success or failure of these measures has been anecdotal in nature without the advantage of controlled clinical trials to identify the best approach to management.

Principles of management of leg ulcers include education, protection, infection control, debridement, and compression bandages. Debridement could be surgical, medical, or biological. Osteomyelitis may complicate chronic leg ulcers, especially those with deep wounds, and it is advisable to rule out this complication with a bone scan or MRI and bone biopsy if needed. Rarely, a leg ulcer is the cause of systemic infections, and occasional deaths attributable to sepsis secondary to a leg ulcer infection have been reported. Also exceedingly rare is the need for amputation of the leg or the foot affected by the ulcer.

Recent advances in the management of leg ulcers include topical applications of analgesics, including opioids for pain, topical application of a platelet-derived growth factor prepared either autologously (Procuren) or by recombinant technology (Regranex), and the use of cultured skin grafts. The use of semipermeable polymeric membrane dressing seems to promote healing in diabetic foot ulcers. Moreover, manuka honey (from New Zealand) has been reported to heal diabetic foot ulcers.

The role of hydroxyurea in the treatment of sickle leg ulcers is presently unclear, however, the weight of evidence suggests the hydroxyurea does not cause, prevent, or speed healing of ulcers in SCD. Chronic transfusion has been advocated for treatment of sickle leg ulcers; however, there is no systematic or controlled data that shows its efficacy. Although it is common for the ulcer to heal when $\mathrm{D}$ transfusion therapy is initiated, they often recur when it is stopped.

\section{Prevention}


Old studies of nutritional deficiencies in SCD demonstrated that zinc deficiency is common in individuals with SCD. The mineral is thought to play an important role in immune function and wound healing. Early, small but randomized studies showed that administration of zinc sulfate $220 \mathrm{mg}$ three times a day resulted in more rapid healing of leg ulcers [10].

Clinical observations and epidemiological studies document the important role of local trauma in the onset of leg ulcers. This provides a number of simple interventions that can be taught to older children and young adults, which will potentially reduce the prevalence of this troubling complication, such as protective legging with nonelastic lower extremity orthoses with ankle straps, to be worn during working hours. Educational interventions should be targeted to health professionals so that the lower extremities are avoided as sites for venipuncture and vascular access unless absolutely necessary. Although not all studies support the importance of venous stasis in the development of leg ulcers, the most systematic epidemiological studies and clinical experience suggest that this may be a major determinant of the success of treatment and prevention. Control of venous stasis requires behavioral modification, may result in need for occupational rehabilitation, and may be common to all successful interventions. Venous stasis may also explain the causal link between pulmonary hypertension and occurrence of leg ulcers. Bed rest and in general limiting the amount of standing time seems to have an impact on wound healing as well.

Finally, epidemiological studies from Jamaica suggest that socioeconomic status has a major effect on the frequency of leg ulcers in their cohort study.

\section{Linkage of Leg Ulcers to Hemolysis-Associated Pulmonary Hypertension in Adults with SCD}

Recent evidence suggests that patients with SCD and leg ulcers may be also at risk to develop other, much more serious disease complications such as pulmonary hypertension, defined by tricuspid regurgitant velocity (TRV), and high NT-proBNP levels, priapism and, possibly renal disease. Taylor et al. analyzed a large sample of patients, which included the NIH data base and the cooperative study of SCD (CSSD) database and found that hyperhemolysis subjects, defined by the top quartile of serum LDH level, had a higher prevalence of leg ulcers (OR $3.27,95 \% \mathrm{Cl}$ $1.92-5.53, P<0.0001$ ), priapism (OR 2.62, 95\% Cl 1.13-6.90, $P=0.03$ ), and pulmonary hypertension (OR 4.32, 95\% Cl 2.12-8.60, $P<0.0001$ ), whereas osteonecrosis (OR 0.32, 95\% $\mathrm{Cl} 0.19-0.54, P<0.0001$ ) and pain (OR 0.23 , $95 \% \mathrm{Cl} 0.09-0.55, P=0.0004$ ) were less prevalent [11]. Hyperhemolysis was influenced by $\mathrm{HbF}$ and alfa thalassemia and was a risk factor for early death in the larger CSSCD population (Hazard Ratio $=1.97, P=0.02$ ). De Castro et al. reported that $37 \%$ of $\mathrm{HbSS}$ patients with pulmonary hypertension defined by TRV had an history of chronic leg ulcers compared with $15 \%$ of those without increased TRV [12]. More recently, a Turkish group reported on the results of Echo Doppler analysis on of $88 \mathrm{HbSS}$ patients; 12 patients (10 males, 2 females) had active ulcer or healed scar (group I) and 76 patients (40 males, 36 females) had no active leg ulcer or history of (group II). The prevalence of pulmonary hypertension in group I and group II was $91.6 \%(n=11)$ and $31.6 \%(n=24)$, respectively $(P$ 
$=0.0001)$. Patients with leg ulceration had increased left atrium and right ventricular diameters at diastole and also had increased left ventricular end-diastolic and end-systolic diameters. They concluded that patients with HbSS, especially those with leg ulcers should be screened for pulmonary hypertension.

We have recently examined an NIH cohort of almost 400 adult patients and found that $18 \%$ of all subjects had a history of leg ulceration (21.4\% of $\mathrm{HbSS}$ and $9.6 \%$ of $\mathrm{HbSC}$ patients). Table I Patients affected were older, had markers of significantly more severe hemolysis, high serum uric acid, alkaline phosphatase, NT-proBNP, and lower serum albumin. They had a significantly higher prevalence of elevated TRV, suggestive of pulmonary hypertension (Fig. 1). Significantly, patients with a history of leg ulcers were more likely to have died by the time of data analysis ( $21 \%$ vs. $9 \%, P=0.02$ ), with a similar trend on Kaplan-Meier analysis (Fig. 2 ). None of the other parameters evaluated were significantly associated with leg ulceration, including history of pain, acute chest syndrome, stroke, or priapism. Patients on hydroxyurea therapy or chronic transfusions did not have a lower incidence of leg ulcers, and

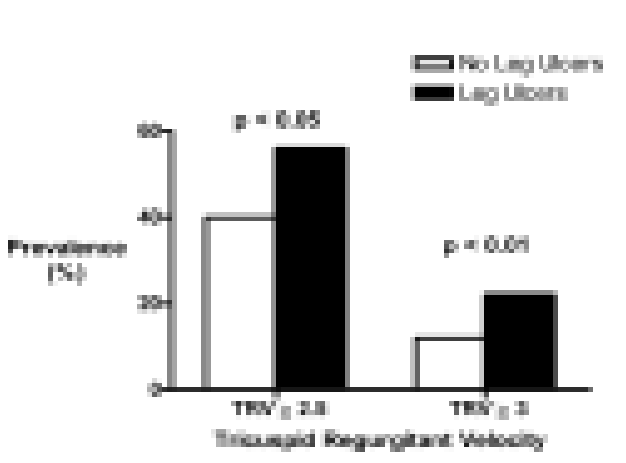
$\mathrm{HbF}$ was not protective.

Figure 1. Prevalence of elevated TRV among study participants by history of leg ulcers. $P$ value from Chi-square test of independence between grouped TRV and history of leg ulcers (yes/no).

Table I. Characteristics of Study Participants by History of Leg Ulcers, NIH Sickle Cell Cohort

No leg ulcers

$N \quad$ Median (IQR) $)^{\mathrm{a}} \quad N \quad$ Median (IQR)

History of leg ulcers
$P$ Value $^{\text {b }}$

Characteristic

\begin{tabular}{|c|c|c|c|c|c|c|}
\hline Age (years) & & 325 & $31(25-42)$ & 71 & $42(32-49)$ & $<0.0001$ \\
\hline \multirow{2}{*}{ Chronic transfusions } & Yes & 118 & & 30 & & \multirow{2}{*}{0.3} \\
\hline & No & 171 & & 32 & & \\
\hline Hydroxyurea treatment, $N(\%)$ & & 317 & $123(39 \%)$ & 71 & 25 (35\%) & 0.6 \\
\hline \multirow{2}{*}{$\operatorname{TRV}(\mathrm{m} / \mathrm{sec})$} & $\geq 2.5$ & 122 & & 38 & & 0.02 \\
\hline & $>2.9$ & 37 & & 15 & & 0.006 \\
\hline
\end{tabular}


Table I. Characteristics of Study Participants by History of Leg Ulcers, NIH Sickle Cell Cohort

$$
\text { No leg ulcers History of leg ulcers }
$$

\begin{tabular}{lccccc}
\cline { 2 - 5 } Characteristic & $N$ & Median (IQR) & $N$ & Median (IQR) $^{\mathrm{a}}$ & $P$ Value $^{\mathrm{b}}$ \\
\hline Oxygen saturation (\%) & 242 & $98(96-99)$ & 45 & $96(92-98)$ & 0.01 \\
Hemoglobin (g/dL) & 309 & $9.8(8.5-10.9)$ & 69 & $8.2(7.3-9.2)$ & $<0.0001$ \\
Reticulocyte count $\left(\times 10^{-3} / \mathrm{ML}\right)$ & 299 & $207(136-313)$ & 68 & $278(160.5-404)$ & 0.003 \\
Lactate dehydrogenase (U/L) & 279 & $311(288-405)$ & 64 & $371.5(274-497.5)$ & 0.004 \\
Aspartate aminotransferase (U/L) & 306 & $35(25-49)$ & 68 & $41.5(31.5-54)$ & 0.02 \\
Albumin (g/dL) & 307 & $4.2(3.8-4.4)$ & 69 & $4.0(3.7-4.2)$ & 0.001 \\
Alkaline phosphatase (IU/L) & 308 & $87(66-117)$ & 68 & $97(79-140)$ & 0.02 \\
Blood urea nitrogen (mg/dL) & 308 & $7(5-10)$ & 68 & $8(6-15.5)$ & 0.01 \\
Uric acid (mg/dL) & 307 & $5.6(4.5-7.0)$ & 69 & $6.8(4.8-8.3)$ & 0.0004 \\
NT-proBNP & 259 & $58(29-131)$ & 59 & $132(77-321)$ & $<0.0001$ \\
Deaths, $N(\%)$ & 295 & $25(8.5)$ & 68 & $14(20.6)$ & \\
\hline
\end{tabular}

- a Except where otherwise noted.

- b From Wilcoxon rank-sum test or Pearson Chi-square statistic between history of leg ulcer groups.

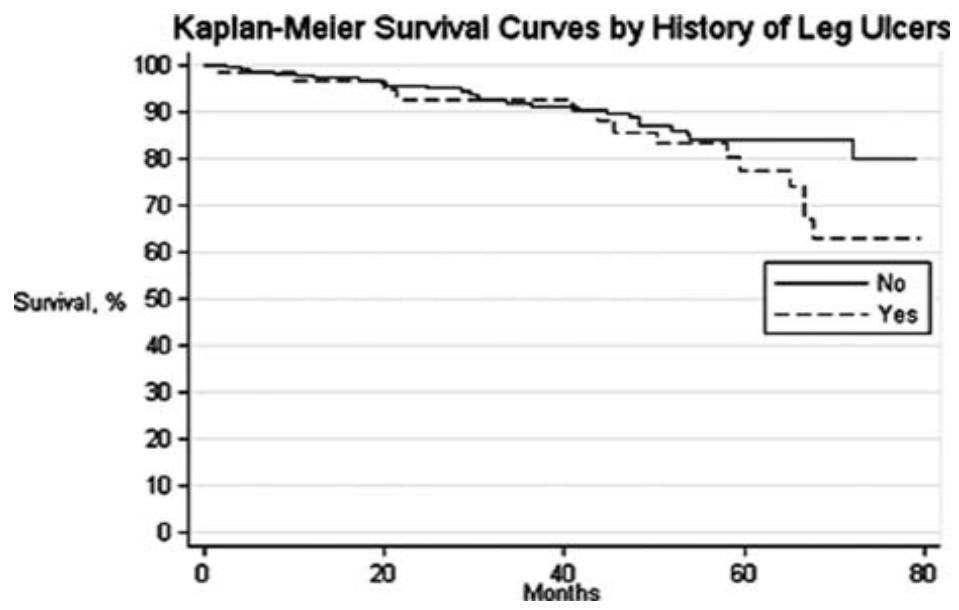

Figure 2. Kaplan-Meier survival curve by history of leg ulcers.

From this data, it is reasonable to conclude that sickle cell leg ulcers are a marker of disease severity. In patients without SCD, high uric acid is identified as a possible cause of hypertension and a marker of risk for cardiovascular 
disease, pulmonary hypertension, and early mortality. The epidemiological relationship between leg ulcers and pulmonary hypertension supports an overlap of pathobiological mechanisms.

\section{Genetic Modifiers of Sickle Cell Leg Ulcers}

Twin studies have shown strong heritability of venous functions in humans and suggest that there may be genetic modifiers that modulate the risk for leg ulcers in patients with sickle cell anemia [13]. This hypothesis is consistent with the large patient to patient variability in developing this complication. However, the literature on genetic associations with leg ulcer is very limited. We review results from candidate gene studies and then report preliminary results of a genome-wide association study (GWAS) for an unbiased discovery of genetic modifiers of this phenotype.

\section{Candidate Gene Studies}

Limited results are available linking susceptibility to leg ulcer and genetic variants. A small study of 9 cases and 29 controls showed possible associations of HLA-B35 and CW14 alleles: the study showed that carriers of both alleles increase the risk for the complication by 17 fold [14]. This study however was very small, and the region identified is very difficult to study because of the long range linkage disequilibrium.

In a candidate gene study of 243 cases (aged $34.8 \pm 12.5$ years) and 516 controls (aged $30.7 \pm 9.4$ years) from the CSSCD, Nolan et al. examined the associations of 250 single nucleotide polymorphisms (SNPs) in 60 candidate genes that were selected for their putative role in the pathophysiology of sickle cell anemia [15]. The analysis found associations with SNPs in Klotho, TEK, and several genes in the TGF- $\beta$ /BMP signaling pathway by genotypic association analyses [15]. KL directly or indirectly promotes endothelial NO production and the TEK receptor tyrosine kinase is involved in angiogenesis. The TGF- $\beta / B M P$ signaling pathway modulates wound healing and angiogenesis, among its other functions. Some of the same SNPs were also associated with risk for stroke, priapism and pulmonary hypertension [16-18]. The results are consistent with the observation that leg ulcer is associated with the intensity of hemolytic anemia, and other subphenotypes of HbSS [11].

\section{Genome-Wide Association Study}

In the past few years, high-throughput technology has changed the field of human genetics. Commercial arrays can be used to genotype up to 2M SNPs simultaneously and they allow for an unbiased screening of the a large number of variants of the human genome [19]. We recently completed a GWAS of leg ulcer in almost 1,400 sickle cell anemia patients from the CSSCD that included 219 leg ulcer cases and 1180 disease free subjects. We used innovative statistical techniques to discover robust associations of both individual SNPs and genes with leg ulcer [19]. Single SNP analysis identified 30 SNPs that are associated with leg-ulcer after we adjusted the analysis by age and gender. The results included SNPs in ALCAM, supposed to regulate endothelial functions, and several SNPs in gene desert regions. These associations with variants in gene-desert regions suggested that gene regulation may be involved with the disease. 
Because the study is underpowered, we also used a SNP-set analysis that, rather than looking at the association of one SNP at a time, looks at the global associations of a set of SNPs. We showed that this approach has better power to capture associations that individually do not meet genome-wide significance, but can point to region of interest when they are analyzed as a group [20]. This analysis identified several genes that appear to be globally associated with leg ulcer, and the full analysis will be reported elsewhere. Interestingly, this analysis showed that a cluster of genes in the major histocompatibility complex class III region in chromosome 6 are strongly associated with leg ulcer. This region is in strong linkage disequilibrium with the HLA-B complex that was linked to leg ulcers in the candidate gene analysis of Ofosu et al. [14]. Furthermore, the same group of genes was found associated with increased susceptibility to venous leg ulcer in older subjects who do not have SCD [14, 21].

\section{Conclusions}

These results are very preliminary and need to be followed by replication in independent studies to distinguish between true positive and false positive associations, and then resequencing to target the functional variants that can be used for treatment and development of risk prediction models. Because SCD is a rare disease in developed countries, it is very difficult to find independent studies that can be used for replication and have a sufficiently large sample size. A consortium of sickle cell anemia centers interested in genetic analysis, the SickleGen Consortium, is being developed, to facilitate future collaborations.

\section{References}

1. HerrickJB.Peculiar elongated and sickle-shaped red blood corpuscles in a case of severe anemia. 1910. Yale J Biol Med 2001;74:179-184.

2. DiggsLW,ChingRE.Pathology of sickle cell anemia. South Med J 1934;27:839-845.

3. SerjeantGR.Leg ulceration in sickle cell anemia.Arch Intern Med 1974;133:690-694.

4. AkinyanjuO,Akinsetel.Leg ulceration in sickle cell disease in Nigeria. Trop Geogr Med 1979;31:87-91.

5. KoshyM,EntsuahR,KorandaA, et al.Leg ulcers in patients with sickle cell disease.Blood 1989;74:1403-1408.

6. MackAK,KatoGJ.Sickle cell disease and nitric oxide: A paradigm shift? Int J Biochem Cell Biol 2006;38:1237-1243.

7. MohanJS,MarshallJM,ReidHL, et al.Postural vasoconstriction and leg ulceration in homozygous sickle cell disease.Clin Sci (Lond) 1997;92:153-158.

8. TrentJT,KirsnerRS.Leg ulcers in sickle cell disease.Adv Skin Wound Care 2004;17:410-416.

9. SerjeantGR,SerjeantBE,MohanJS,ClareA.Leg ulceration in sickle cell disease: Medieval medicine in a modern world. Hematol Oncol Clin North Am 2005;19:943-956, viii-ix. 
10. SerjeantGR,GallowayRE,GueriMC.Oral zinc sulphate in sickle-cell ulcers.Lancet 1970;2:891-892.

11. TaylorJGt,NolanVG,MendelsohnL, et al.Chronic hyper-hemolysis in sickle cell anemia: association of vascular complications and mortality with less frequent vasoocclusive pain.PloS one 2008;3:e2095.

12. De CastroLM,JonassaintJC,GrahamFL,Ashley-KochA,TelenMJ.Pulmonary hypertension associated with sickle cell disease: Clinical and laboratory endpoints and disease outcomes. Am J Hematol 2008;83:19-25.

13. BrinsukM,TankJ,LuftFC,BusjahnA,JordanJ.Heritability of venous function in humans.Arterioscler Thromb Vasc Biol 2004;24:207-211.

14. OfosuMD,CastroO,AlarifL.Sickle cell leg ulcers are associated with HLA-B35 and Cw4.Arch Dermatol 1987;123:482-484.

15. NolanVG,AdewoyeA,BaldwinC, et al.Sickle cell leg ulcers: Associations with haemolysis and SNPs in Klotho, TEK and genes of the TGF-beta/BMP pathway.Br J Haematol 2006;133:570-578.

16. SebastianiP,RamoniMF,NolanV, et al.Genetic dissection and prognostic modeling of overt stroke in sickle cell anemia. Nat Genet 2005;37:435-440.

17. NolanVG,BaldwinC,MaQ, et al.Association of single nucleotide polymorphisms in klotho with priapism in sickle cell anaemia.Br J Haematol 2005;128:266-272.

18. Ashley-KochAE,ElliottL,KailME, et al.Identification of genetic polymorphisms associated with risk for pulmonary hypertension in sickle cell disease.Blood 2008;111:5721-5726.

19. SebastianiP,TimofeevN,DworkisDA, et al.Genome-wide association studies and the genetic dissection of complex traits. Am J Hematol 2009;84:504-515.

20. SebastianiP,SolovieffN,HartleySW, et al.Genetic modifiers of the severity of sickle cell anemia identified through a genome-wide association study.Am J Hematol 2010;85:29-35.

21. WallaceHJ,VandongenYK,StaceyMC.Tumor necrosis factor-alpha gene polymorphism associated with increased susceptibility to venous leg ulceration.J Invest Dermatol 2006;126:921-925. 